Chinese Journal of Organic Chemistry

ARTICLE

\title{
可见光催化分子内环芳构化合成 10-菲酚衍生物
}

\author{
滕巧玲 ${ }^{a}$ 许露露 ${ }^{a}$ 程冬萍 $*, b$ 许孝良 $*, a$ \\ ( ${ }^{a}$ 浙江工业大学化学工程学院 杭州 310014) \\ ( ${ }^{b}$ 浙江工业大学药学院 杭州 310014)
}

\begin{abstract}
摘要 菲衍生物在药物化学和材料科学中具有重要的地位. 可见光催化具有绿色、反应条件温和、应用潜力大等优点, 已经逐渐成为有机合成中的有力工具. 报道了以 $\operatorname{Ir}\left[\mathrm{dF}\left(\mathrm{CF}_{3}\right) \mathrm{ppy}\right]_{2}(\mathrm{dtbbpy}) \mathrm{PF}_{6}$ 为可见光催化剂, 2-芳基苯甲酰乙酸衍生物 发生分子内环芳构化反应，以中等至良好的收率得到一系列 10-菲酚衍生物. 此外, 对该反应的机理进行了推测。

关键词 可见光催化剂; 自由基反应; 分子内环芳构化; 菲酚
\end{abstract}

\section{Synthesis of 10-Phenanthrenol Derivatives via Visible Light Catalyzed Itramolecular Cycloaromatization}

\author{
Teng, Qiaoling ${ }^{a} \quad \mathrm{Xu}$, Lulu $^{a} \quad$ Cheng, Dongping $^{*, b} \quad \mathrm{Xu}$, Xiaoliang ${ }^{*, a}$ \\ $\left({ }^{a}\right.$ College of Chemical Engineering, Zhejiang University of Technology, Hangzhou 310014) \\ $\left({ }^{b}\right.$ College of Pharmaceutical, Zhejiang University of Technology, Hangzhou 310014)
}

\begin{abstract}
Phenanthrene derivatives play an important role in pharmaceutical chemistry and material science. Due to its advantages of green, mild reaction conditions and great application potential, visible light catalysis has become a powerful tool in organic synthesis. In this paper, under the catalysis of photocatalyst $\operatorname{Ir}\left[\mathrm{dF}\left(\mathrm{CF}_{3}\right) \text { ppy }\right]_{2}(\mathrm{dtbbpy}) \mathrm{PF}_{6}$, a series of 10 -phenanthrenol derivatives were synthesized from 2-arylbenzoyl acetate derivatives in moderate to good yields through intramolecular cycloaromatization. In addition, the plausible reaction mechanism was also proposed.
\end{abstract}

Keywords visible-light photocatalysis; radical reaction; intramolecular cycloaromatization; phenanthrenols

菲衍生物广泛存在于自然产物中, 是一类重要的多 环芳烃化合物 ${ }^{[1]}$. 由于具有抗癌 ${ }^{[2]} 、$ 抗症疾 ${ }^{[3]}$ 、抗过敏 ${ }^{[4]}$ 、 抗菌 ${ }^{[5]}$ 等多种生物活性, 菲衍生物在药物化学研究中备 受关注. 此外, 该类化合物还具有显著的光导、光化学 和电致发光性质, 使其成为材料科学中常见的结构基 序 $^{[6]}$. 在过去几十年中, 菲衍生物的合成方法得到了不 断发展, 大致可分为两类. 第一类主要是以二苯乙烯衍 生物为起始原料发生分子内环烷化反应 ${ }^{[7]}$ 、缩合反应 ${ }^{[8]}$ 、 光化学环化反应 ${ }^{[9]}$ 等得到目标产物. 其中 Mallory 反应 是以二苯乙烯衍生物发生氧化光环化制备菲衍生物最 常用的方法之一, 该反应涉及使用催化量的碘氧化二氢 菲中间体生成菲的过程 ${ }^{[10]}$. 另外顺式 2-碘二苯乙烯的自 由基环化 ${ }^{[11]}$ 和双 ( $N$-对甲苯磺酰腙)的卡宾二聚反应 ${ }^{[12]}$ 也 是合成菲衍生物的有效方法. 第二种是以联苯衍生物为
起始原料来合成, 如 2,2'-二羰基联苯的 McMurry 反 应 $^{[13]} 、 2,2^{\prime}$-二乙烯基联苯的烯烃复分解反应 ${ }^{[14]}$ 、联苯与 内炔的分子间环加成反应 ${ }^{[15]}$ 等. 此外以 2-乙炔基联苯衍 生物为起始原料, 通过过渡金属催化 ${ }^{[16]}$ 、碱催化 ${ }^{[17]}$ 、亲 电环化 ${ }^{[18]}$ 合成菲衍生物的反应也得到了广泛研究. 尽 管上述方法可以有效合成菲衍生物，但是有些反应仍然 存在效率低、区域选择性差、反应条件苛刻等缺点. 鉴 于菲衍生物在许多领域中的重要性, 开发更方便、更温 和、更高效的菲衍生物制备方法仍然具有重要意义.

近几年来, 可见光光催化因其符合绿色化学的要求 取得了巨大的研究进展 ${ }^{[19]}$, 已经被成功地应用于脱羧、 脱嗍、烷基化、环加成、还原、氧化、聚合等反应. 最 近 Wang 等 ${ }^{[20]}$ 报道了 $N$-溴代丁二酰亚胺(NBS)诱导的 2芳基苯甲酰乙酸衍生物发生分子内环化反应. 该反应为

\footnotetext{
* Corresponding authors. E-mail: chengdp@zjut.edu.cn; xuxiaoliang@zjut.edu.cn Received May 28, 2020; revised July 21, 2020; published online August 5, 2020 Project supported by the Zhejiang Provincial Natural Science Foundation (Nos. LY18B020018, LY15B020004) and the National Natural Science Foundation of China (No. 21602197).

浙江省自然科学基金(Nos. LY18B020018, LY15B020004)和国家自然科学基金(No. 21602197)资助项目.
} 
自由基历程, 需要使用过量的氧化剂叔丁基过氧化氢 (TBHP). 基于我们长期以来对可见光催化反应的关 注 ${ }^{21]}$, 在此报道可见光催化 2-芳基苯甲酰乙酸衍生物发 生分子内环芳构化反应, 生成一系列的 10-菲酚衍生物.

\section{1 结果与讨论}

首先, 选择 2-苯基苯甲酰乙酸乙酯(1a)作为模型底 物, 以 $\operatorname{Ir}\left[\mathrm{dF}\left(\mathrm{CF}_{3}\right) \mathrm{ppy}\right]_{2}(\mathrm{dtbbpy}) \mathrm{PF}_{6}$ 为可见光催化剂, $\mathrm{O}_{2}$ 为氧化剂, $N$-甲基吡咯烷酮(NMP)为溶剂, 在 LED 蓝光 照射下进行反应(表 1, Entry 1), 成功地分离得到目标产 物 2a, 尽管产率只有 $18 \%$. 接着考察了不同可见光催化 剂对反应的影响(Entries 2 4). 当使用 $\mathrm{Ru}(\mathrm{bpy})_{3}\left(\mathrm{PF}_{6}\right)_{2}$ 、 曙红 $Y 、 \operatorname{Ir}(\mathrm{ppy})_{3}$ 作为可见光催化剂时, 通过薄层色谱 (TLC)检测发现原料未减少, 基本没有产物生成, 这可 能与可见光催化剂的氧化还原电位有关. 考虑反应中加 入碱是否能提高收率, 我们改变反应条件为以 $\mathrm{Na}_{2} \mathrm{CO}_{3}$ 为碱, $\mathrm{Na}_{2} \mathrm{~S}_{2} \mathrm{O}_{8}$ 为氧化剂, $N, N$-二甲基甲酰胺(DMF)为溶
剂，在氮气保护下进行反应，以 20\%的收率得到目标产 物 2a (Entry 5). 在篎选的单一溶剂中, 以甲苯或乙醇为 溶剂时收率较高. 考虑到反应中添加剂的溶解度，还篮 选了混合溶剂, 当甲苯/乙醇 $(V: V=5: 1)$ 用作溶剂时, 反应以 $33 \%$ 的收率得到产物 2a (Entries 6 10). 当把反 应中的氧化剂换成 $\mathrm{K}_{2} \mathrm{~S}_{2} \mathrm{O}_{8}$ 或 $\left(\mathrm{NH}_{4}\right)_{2} \mathrm{~S}_{2} \mathrm{O}_{8}$ 时, 收率并没有 得到提高(Entries 11 12). Wang 等报道的工作表明少量 的 NBS 可以诱导 2-芳基苯甲酰乙酸衍生物实现分子内 环芳构化. 当在反应中添加少量的 NBS, 反应收率略微 提升至 41\% (Entry 13), NBS 在可见光催化体系下并没 有起到好的效果. 令人惊喜的是, 当添加催化量的 $\mathrm{KI}$ 时, 反应以 75\%的收率获得 $\mathbf{2 a}$ (Entries 14 16). 随后对 碱进行了篮选, 结果表明 $\mathrm{K}_{2} \mathrm{HPO}_{4}$ 在反应中效果最佳, 2a 的收率可以达到 79\% (Entries 17～19). 控制实验表 明反应在黑暗条件下无产物 $\mathbf{2 a}$ 生成, 证明可见光对该 反应是必不可少的(Entry 20). 当反应体系不加可见光 催化剂时, 产物 2a 的收率降至 22\% (Entry 21). 最终确

表 1 反应条件优化 ${ }^{a}$

Table 1 Optimization of the reaction conditions

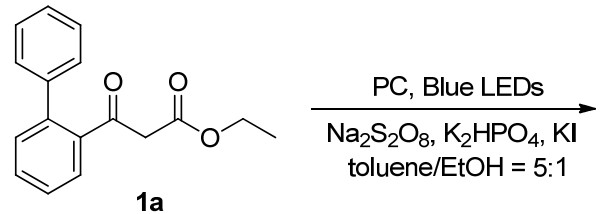<smiles>CCOC(=O)c1c(O)c2ccccc2c2ccccc12</smiles>

2a

\begin{tabular}{|c|c|c|c|c|c|c|}
\hline Entry & Photocatalyst & Oxidant & Base & Additives & Solvent & Yield $^{b} / \%$ \\
\hline $1^{f, g}$ & PC(1) & $\mathrm{O}_{2}$ & - & - & NMP & 18 \\
\hline $2^{\mathrm{e}, f, g}$ & $\operatorname{PC}(2)$ & $\mathrm{O}_{2}$ & - & - & NMP & - \\
\hline $3^{f, g}$ & $\mathrm{PC}(3)$ & $\mathrm{O}_{2}$ & - & - & NMP & Trace \\
\hline $4^{e, f, g}$ & PC(4) & $\mathrm{O}_{2}$ & - & - & NMP & - \\
\hline $5^{g}$ & PC(1) & $\mathrm{Na}_{2} \mathrm{~S}_{2} \mathrm{O}_{8}$ & $\mathrm{Na}_{2} \mathrm{CO}_{3}$ & - & DMF & 20 \\
\hline $6^{g}$ & PC(1) & $\mathrm{Na}_{2} \mathrm{~S}_{2} \mathrm{O}_{8}$ & $\mathrm{Na}_{2} \mathrm{CO}_{3}$ & - & Toluene & 22 \\
\hline $7^{g}$ & PC(1) & $\mathrm{Na}_{2} \mathrm{~S}_{2} \mathrm{O}_{8}$ & $\mathrm{Na}_{2} \mathrm{CO}_{3}$ & - & EtOH & 25 \\
\hline $8^{g}$ & PC(1) & $\mathrm{Na}_{2} \mathrm{~S}_{2} \mathrm{O}_{8}$ & $\mathrm{Na}_{2} \mathrm{CO}_{3}$ & - & Toluene/EtOH $(V: V=1: 1)$ & 27 \\
\hline $9^{g}$ & PC(1) & $\mathrm{Na}_{2} \mathrm{~S}_{2} \mathrm{O}_{8}$ & $\mathrm{Na}_{2} \mathrm{CO}_{3}$ & - & Toluene/EtOH $(V: V=2: 1)$ & 25 \\
\hline $10^{g}$ & PC(1) & $\mathrm{Na}_{2} \mathrm{~S}_{2} \mathrm{O}_{8}$ & $\mathrm{Na}_{2} \mathrm{CO}_{3}$ & - & Toluene/EtOH $(V: V=5: 1)$ & 33 \\
\hline $11^{g}$ & PC(1) & $\mathrm{K}_{2} \mathrm{~S}_{2} \mathrm{O}_{8}$ & $\mathrm{Na}_{2} \mathrm{CO}_{3}$ & - & Toluene/EtOH $(V: V=5: 1)$ & 25 \\
\hline $12^{g}$ & PC(1) & $\left(\mathrm{NH}_{4}\right)_{2} \mathrm{~S}_{2} \mathrm{O}_{8}$ & $\mathrm{Na}_{2} \mathrm{CO}_{3}$ & - & Toluene/EtOH $(V: V=5: 1)$ & 18 \\
\hline $13^{c}$ & PC(1) & $\mathrm{Na}_{2} \mathrm{~S}_{2} \mathrm{O}_{8}$ & $\mathrm{Na}_{2} \mathrm{CO}_{3}$ & NBS & Toluene/EtOH $(V: V=5: 1)$ & 41 \\
\hline 14 & PC(1) & $\mathrm{Na}_{2} \mathrm{~S}_{2} \mathrm{O}_{8}$ & $\mathrm{Na}_{2} \mathrm{CO}_{3}$ & $\mathrm{NaI}$ & Toluene/EtOH $(V: V=5: 1)$ & 35 \\
\hline 15 & PC(1) & $\mathrm{Na}_{2} \mathrm{~S}_{2} \mathrm{O}_{8}$ & $\mathrm{Na}_{2} \mathrm{CO}_{3}$ & KI & Toluene/EtOH $(V: V=5: 1)$ & 75 \\
\hline 16 & PC(1) & $\mathrm{Na}_{2} \mathrm{~S}_{2} \mathrm{O}_{8}$ & $\mathrm{Na}_{2} \mathrm{CO}_{3}$ & $\mathrm{NH}_{4} \mathrm{I}$ & Toluene/EtOH $(V: V=5: 1)$ & 56 \\
\hline 17 & PC(1) & $\mathrm{Na}_{2} \mathrm{~S}_{2} \mathrm{O}_{8}$ & $\mathrm{~K}_{2} \mathrm{HPO}_{4}$ & KI & Toluene/EtOH $(V: V=5: 1)$ & 79 \\
\hline 18 & PC(1) & $\mathrm{Na}_{2} \mathrm{~S}_{2} \mathrm{O}_{8}$ & $\mathrm{~K}_{3} \mathrm{PO}_{4}$ & KI & Toluene/EtOH $(V: V=5: 1)$ & 37 \\
\hline 19 & PC(1) & $\mathrm{Na}_{2} \mathrm{~S}_{2} \mathrm{O}_{8}$ & $\mathrm{NaOAc}$ & KI & Toluene/EtOH $(V: V=5: 1)$ & 49 \\
\hline $20^{d, e}$ & PC(1) & $\mathrm{Na}_{2} \mathrm{~S}_{2} \mathrm{O}_{8}$ & $\mathrm{~K}_{2} \mathrm{HPO}_{4}$ & KI & Toluene/EtOH $(V: V=5: 1)$ & - \\
\hline 21 & - & $\mathrm{Na}_{2} \mathrm{~S}_{2} \mathrm{O}_{8}$ & $\mathrm{~K}_{2} \mathrm{HPO}_{4}$ & KI & Toluene/EtOH $(V: V=5: 1)$ & 22 \\
\hline
\end{tabular}

${ }^{a}$ Reaction conditions: 1a $(0.3 \mathrm{mmol})$, oxidant $(1.2$ equiv.), base (1.2 equiv.), iodide $(5 \mathrm{~mol} \%)$, photocatalyst $(1 \mathrm{~mol} \%)$, solvent $(3.0 \mathrm{~mL})$, reaction time $(12 \sim 48 \mathrm{~h}), 45$ $\mathrm{W}$ blue LEDs irradiation under $\mathrm{N}_{2}$ atmosphere at ambient temperature. $\mathbf{P C}(\mathbf{1})$ : $\operatorname{Ir}\left[\mathrm{dF}\left(\mathrm{CF}_{3}\right) \text { ppy }\right]_{2}(\mathrm{dtbbpy}) \mathrm{PF}_{6}:$ 4,4'-di-tert-butyl-2,2'-bipyridine)bis[3,5-difluoro2-(5-trifluoromethyl-2-pyridinyl-kN)phenyl-kC]iridium(III) hexafluorophosphate; $\mathbf{P C}(2)$ : $\operatorname{Ru}(\mathrm{bpy})_{3}(\mathrm{PF})_{2}$ : tris(2,2'-bipyridine)ruthenium(II) hexafluorophosphate; PC(3): Eosin Y: 2',4',5',7'-tetrabromo-3',6'-dihydroxyspiro(isobenzofuran-1(3H),9'-(9H)xanthen)-3-one; PC(4): $\operatorname{Ir}(\mathrm{ppy})$ : : tris(2-phenylpyridine)iridium(III). ${ }^{b}$ Isolated yields by silica gel column chromatography. ${ }^{c}$ NBS $\left(20\right.$ mol\%). ${ }^{d}$ Without light irradiation. ${ }^{e}$ Substrate 1a remained unreacted. ${ }^{f}$ Without base. ${ }^{g}$ Without additive. 
定了表 1 中的 Entry 17 为最优条件.

在最优化反应条件下，考察了各种取代 2-芳基苯甲 酰乙酸酯及其衍生物在该反应体系中的适用性(表 2). 实验结果表明, $\mathrm{Ar}^{2}$ 环上取代基为吸电子基团或给电子
基团都可以成功发生分子内环芳构化反应，以中等至良 好的收率分离得到相应产物 $(2 \mathrm{~b} \sim 2 \mathrm{p})$. 其中 $\mathrm{Ar}^{2}$ 环的对 位有取代基的底物反应效果最好 $(2 \mathrm{~b} \sim 2 \mathrm{e}$ 和 $\mathbf{2 i} \sim 2 \mathrm{2})$, 可 能由于取代基的电子效应及空间位阻效应对反应的影

表 2 底物拓展 ${ }^{a}$

Table 2 Scope of the substrates<smiles>CC(C)(C)CCC(=O)c1ccccc1-c1ccccc1</smiles>

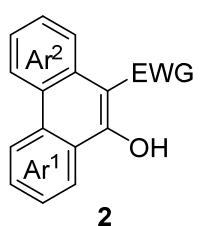<smiles>CCOC(=O)c1c(O)c2ccccc2c2ccccc12</smiles>

2a, 22 h, $79 \%$<smiles>CCOC(=O)c1c(O)c2ccccc2c2c(C)cccc12</smiles>

2f, $48 \mathrm{~h}, 47 \%$<smiles>CCOC(=O)c1c(O)c2ccccc2c2ccc(Cl)cc12</smiles>

2k, 26 h, $70 \%$<smiles>CCOC(=O)c1c(O)c2ccccc2c2c(F)cccc12</smiles>

2o, $30 \mathrm{~h}, 59 \%$<smiles>CCOC(=O)c1c(O)c2cc(F)ccc2c2ccccc12</smiles>

2t, $5 \mathrm{~h}, 85 \%$<smiles>CCOC(=O)c1c(O)c2ccccc2c2ccc(C)cc12</smiles>

2b, 22 h, $77 \%$<smiles>CCOC(=O)c1c(O)c2ccccc2c2c(OC)cccc12</smiles>

2g, 48 h, $47 \%$<smiles>CCOC(=O)c1c(O)c2ccccc2c2ccc(F)cc12</smiles>

2I, $26 \mathrm{~h}, 64 \%$<smiles>CCOC(=O)c1c(O)c2ccccc2c2cc(C)cc(C)c12</smiles>

2p, 30 h, $43 \%$<smiles>CCOC(=O)c1c(O)c2ccc(F)cc2c2ccccc12</smiles>

2u, 6 h, $79 \%$<smiles>CS(=O)(=O)c1c(O)c2ccccc2c2ccccc12</smiles>

2y, 48 h, $0 \%$<smiles>CCOC(=O)c1c(O)c2ccccc2c2ccc(OC)cc12</smiles>

2c, 20 h, $78 \%$<smiles>CCOC(=O)c1c(O)c2ccccc2c2c(C(C)C)cccc12</smiles>

2h, 33 h, $63 \%$<smiles>CCOC(=O)c1c(O)c2ccccc2c2cccc(C)c12</smiles><smiles>CCOC(=O)c1c(O)c2c(c3cc(C)ccc13)=C[C+]=CC=2</smiles>

$2 m+2 m^{\prime}, 22$ h, $48 \%$<smiles>CCOC(=O)c1c(O)c2ccccc2c2cc3ccccc3cc12</smiles>

2q, 46 h, $51 \%$<smiles>CCOC(=O)c1c(O)c2c(F)cccc2c2ccccc12</smiles>

2v, 16 h, $57 \%$<smiles>CCOC(=O)c1c(O)c2ccccc2c2ccc(C(C)(C)C)cc12</smiles>

2d, 20 h, $83 \%$<smiles>CCOC(=O)c1ccc2c(C(=O)OCC)c3ccccc3c(O)c2c1</smiles>

2i, $13 \mathrm{~h}, 77 \%$<smiles>CCOC(=O)c1c(O)c2ccccc2c2c1ccc1ccccc12</smiles>

$2 r^{c}{ }^{c} 26$ h, 33\%<smiles>CCOC(=O)c1c(O)c2cc(OC)ccc2c2ccccc12</smiles>

2w, 39 h, 35\%<smiles>CCOC(=O)c1c(O)c2ccccc2c2ccc(C(C)(C)C)cc12</smiles>

2e, 20 h, $90 \%$

2j, 21 h, 30\%<smiles>CCOC(=O)c1c(O)c2ccccc2c2ccc(C)cc12</smiles>

2n, 48 h, $30 \%$<smiles>O=C(Nc1ccccc1)c1c(O)c2ccccc2c2ccccc12</smiles>

$2 \mathbf{s},{ }^{c} 48 \mathrm{~h}, 32 \%$<smiles>N#Cc1c(O)c2ccccc2c2ccccc12</smiles>

2x, 48 h, $0 \%$

${ }^{a}$ Reaction conditions: 1a $(0.3 \mathrm{mmol}), \mathrm{Na}_{2} \mathrm{~S}_{2} \mathrm{O}_{8}$ (1.2 equiv.), $\mathrm{K}_{2} \mathrm{HPO}_{4}$ (1.2 equiv.), $\mathrm{KI}(5 \mathrm{~mol} \%), \operatorname{Ir}\left[\mathrm{dF}_{(} \mathrm{CF}_{3}\right)$ ppy $]_{2}(\mathrm{dtbbpy}) \mathrm{PF}_{6}(1 \mathrm{~mol} \%)$, toluene/EtOH $(V: V=5: 1$ $(3.0 \mathrm{~mL})$, blue LEDs irradiation under $\mathrm{N}_{2}$ atmosphere at ambient temperature. ${ }^{b}$ Isolated yields by silica gel column chromatography. ${ }^{c} \mathrm{KI}(10 \% \mathrm{~mol})$. 
响. 3,5-二甲基取代的底物仅以 43\%的收率得到 2p. 有 趣的是, 邻位异丙基取代的底物 $\mathbf{1 h}$ 在反应中没有发生 异丙基的裂解, 并以 $63 \%$ 的收率转化为产物 $\mathbf{2 h}$. 值得注 意的是, 乙烯基或甲硫基取代的底物 $1 \mathrm{j}$ 和 $1 \mathrm{n}$ 也可以顺 利发生反应, 均以 $30 \%$ 的收率获得产物. 当 $\mathrm{Ar}^{2}$ 环上为 间甲基取代时，得到 $\mathbf{2 m}$ 和 $\mathbf{2 m}$ '的混合物产率为 $48 \%$, 两 种产物极性相近, 使用柱层析和大板均未分开. $\mathrm{Ar}^{2}$ 环上 为荎基取代的底物 $1 \mathrm{q}$ 和 $1 \mathrm{r}$ 经环芳构化得到多环芳烃化 合物 $\mathbf{2 q}$ 和 $\mathbf{2 r}$, 产率分别为 $51 \%$ 和 33\%. 在标准反应条 件下, $N$-苯酰胺取代底物 $1 \mathrm{~s}$ 可以发生环化反应, 产物 $2 \mathrm{~s}$ 的收率为 $32 \%$. 此外, 在 $\mathrm{Ar}^{1}$ 环的不同位置氟或甲氧基 取代的底物也能顺利反应 $(\mathbf{2 t} \sim \mathbf{2 w})$, 其中对位氟取代底 物 2t 收率最高, 为 $85 \%$. 遗憾的是, 甲磺酰或氰基取代 的底物 $1 \mathrm{x}$ 和 $1 \mathrm{y}$ 在这个反应体系中没有相应的目标产物 生成.

为了初步探索反应机理, 进行了一系列探究实验 (Scheme 1). 首先在反应体系中加入 2 equiv. 2,2,6,6-四 甲基哌啶-1-氧基(TEMPO), 只能得到痕量的产物 $\mathbf{2 a}$, 表明该反应可能涉及自由基历程. 在不添加 $\mathrm{Na}_{2} \mathrm{~S}_{2} \mathrm{O}_{8}$ 、 $\mathrm{KI}$ 和光催化剂的条件下, 往反应体系中加入等物质的 量的 $\mathrm{I}_{2}$, 以 $61 \%$ 的收率分离得到产物 2a. 结果表明, 该 反应可能发生了原位碘化. 令人遗憾的是, 我们无法捕 获或表征反应过程中可能存在的碘化物中间体.<smiles>CCOC(=O)CC(=O)c1ccccc1-c1ccccc1</smiles>

$1 a$<smiles>CCOC(=O)CC(=O)c1ccccc1-c1ccccc1</smiles>

$1 \mathrm{a}$

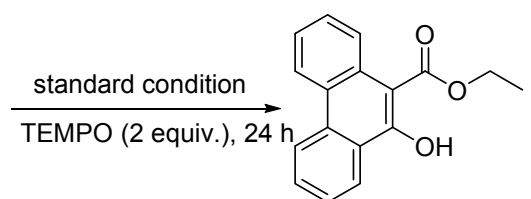

2a, trace

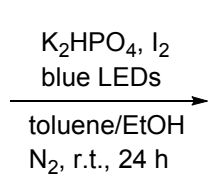<smiles>CCOC(=O)c1c(O)c2ccccc2c2ccccc12</smiles>

2a, $61 \%$
图式 1 机理探究

Scheme 1 Mechanism exploration

根据上述结果和相关文献报道 ${ }^{[20-22]}$, 提出了可能的 反应机理(Scheme 2). Muniz 等 ${ }^{[22]}$ 的工作证实了微量的 水可以引发分子碘发生歧化反应, 快速生成次碘酸盐, 同时 Ishihara 等 ${ }^{[23]}$ 报道的相关工作也表明次碘酸盐具有 催化作用. 由此, 我们推测在本反应中, 催化量的 KI 被 $\mathrm{Na}_{2} \mathrm{~S}_{2} \mathrm{O}_{8}$ 氧化为 $\mathrm{I}_{2}$, 通过歧化反应转化为 HOI 和 HI. 活 化的次碘酸盐引发底物 1a 发生原位碘化, 生成中间体
A. 在蓝光 LED 照射下, $\mathbf{A}$ 发生 $\mathrm{C}-\mathrm{I}$ 键均裂生成 $\mathbf{B}$, 从 而引发自由基循环. B 发生分子内自由基环化得到 C. C

脱质子得到自由基阴离子 $\mathbf{D}$, 随后 $\mathbf{D}$ 被 $\mathbf{A}$ 氧化得到目标 产物 $2 \mathbf{a}$ 和 $[\mathbf{A}]^{\circ-}$. [A] $]^{--}$脱去 $\mathrm{I}^{-}$重新得到 $\mathbf{B}$ (路线 I). 或者 $\mathbf{C}$ 被 $\mathbf{A}$ 氧化生成 $\mathbf{E}$, 随后 $\mathbf{E}$ 脱去质子得到目标产物 $\mathbf{2 a}$ (路 线 II).

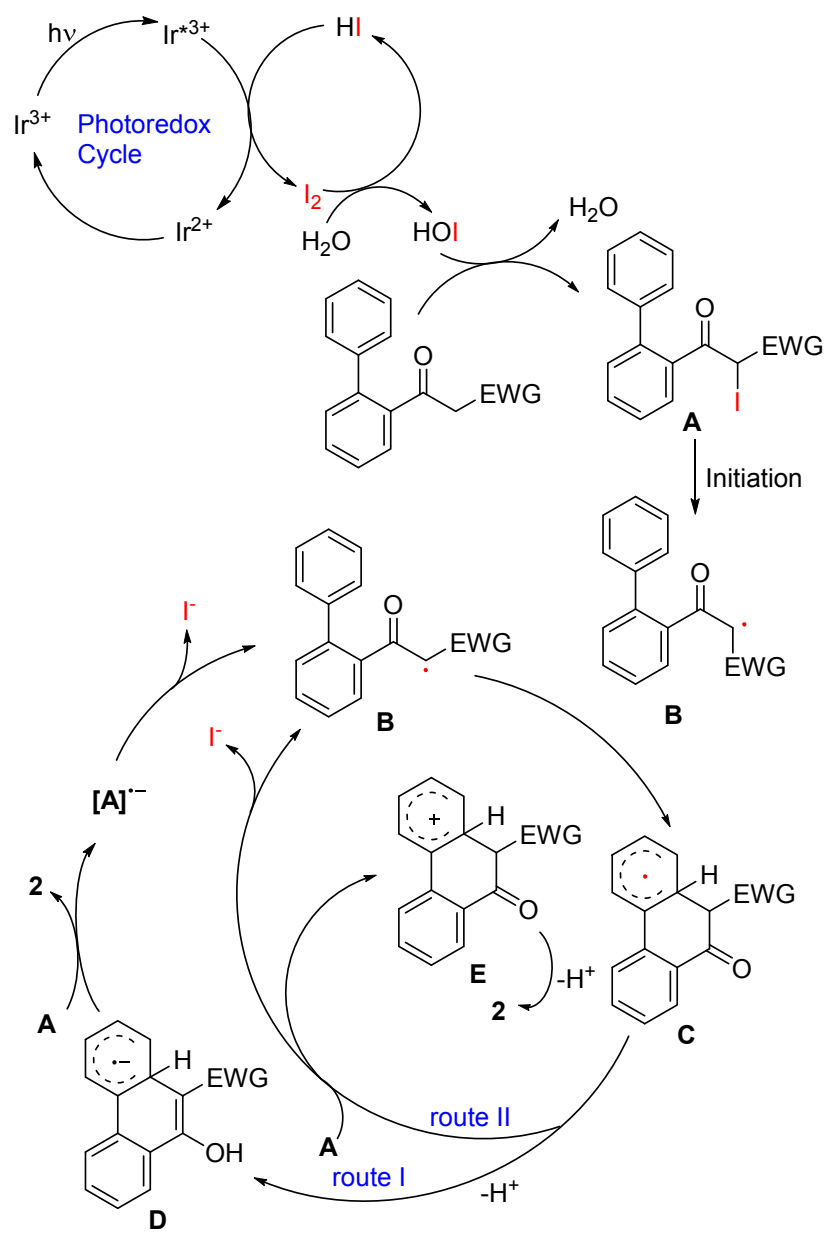

图 2 可能的反应机理

Scheme 2 Plausible reaction mechanism

\section{2 结论}

报道了可见光催化 2-芳基苯甲酰乙酸衍生物的分 子内环芳构化反应, 该反应条件温和, 以中等至良好的 收率合成 10-菲酚衍生物, 为菲衍生物的合成提供一条 路线，同时拓宽可见光催化在有机合成中的应用. 起始 原料联苯衍生物可以通过 Suzuki-Miyaura 偶联反应高效 合成.

\section{3 实验部分}

\section{1 仪器与试剂}

${ }^{1} \mathrm{H}$ NMR 和 ${ }^{13} \mathrm{C}$ NMR 由 Bruker VANCE III (500 $\mathrm{MHz}$ )核磁共振仪测定，内标为四甲基硅烷(TMS), 溶剂 为気代氯仿或氛代二甲基亚砜; ESI 低分辨和高分辨质 谱由 Varian 1200 质谱分析仪测定; 熔点由精松 X-4 显微 
熔点仪测定, 温度计未校正; 其他使用的仪器为 Synthware MS-H-Pro ${ }^{+}$磁力摚拌器、Heidolph ML/HB/G3 旋转蒸发仪、25 mL Schlenk tube.

所有试剂均为市售的分析纯试剂, 直接使用. 甲 苯、过硫酸钠、过硫酸钾、过硫酸铵、磷酸三钾均购于 国药集团化学试剂有限公司, 无水碳酸钠购于太仓美达 试剂有限公司, $N$-溴代丁二酰亚胺购于上海化学试剂采 购供应五联化工厂，碘化铵、磷酸氢二钾购于阿拉丁, 碘化钠购于浙江海川化学品有限公司, 无水乙酸钠购于 温州润华化工实业公司. 柱层析硅胶(200～300 目)购 于青岛鼎康硅胶有限公司, 展开剂使用重蒸工业级石油 醚(PE)和乙酸乙酯(EA). 2-芳基苯甲酰乙酸衍生物(化合 物 1)按照文献方法制备 ${ }^{[24-27]}$

\section{2 化合物 $2 a \sim 2 w$ 的合成}

在氮气保护下, 将化合物 $1(0.3 \mathrm{mmol}) 、 \mathrm{~K}_{2} \mathrm{HPO}_{4}$ (0.36 mmol)、 $\mathrm{Na}_{2} \mathrm{~S}_{2} \mathrm{O}_{8}(0.36 \mathrm{mmol}) 、 \mathrm{KI}$ (0.015 mmol, 5 mol\%)、 $\operatorname{Ir}\left[\mathrm{dF}\left(\mathrm{CF}_{3}\right) \mathrm{ppy}\right]_{2}(\mathrm{dtbbpy}) \mathrm{PF}_{6} \quad(0.003 \mathrm{mmol}, 1$ $\mathrm{mol} \%$ )、甲苯 $(2.5 \mathrm{~mL})$ 和乙醇 $(0.5 \mathrm{~mL})$ 分别加入 $25 \mathrm{~mL}$ 干 燥的 Schlenk 反应管. 将反应混合物放置在距离 $45 \mathrm{~W}$ Blue LED 灯约 $5 \mathrm{~cm}$ 处, 并在室温下搅拌 5 48 h, 用 TLC 板追踪反应. 待反应结束用旋转蒸发仪除去溶剂, 将残渣与硅胶混合干法上柱. 以石油醚/乙酸乙酯 $(V /$ $V=20 / 1)$ 为洗脱剂, 在硅胶 (200 300 目)上进行柱色谱 纯化, 得到纯产物 $\mathbf{2 a} \sim \mathbf{2 w}$.

10-羟基菲-9-羧酸乙酯(2a): $62.8 \mathrm{mg}$, 产率 79\%. 白 色固体, m.p. 103 104 ${ }^{\circ} \mathrm{C}$ (文献值 $\left.{ }^{[20]} 109 \sim 110{ }^{\circ} \mathrm{C}\right) ;{ }^{1} \mathrm{H}$ NMR (500 MHz, $\left.\mathrm{CDCl}_{3}\right) \delta: 13.40(\mathrm{~s}, 1 \mathrm{H}), 8.82 \sim 8.80(\mathrm{~d}$, $J=8.5 \mathrm{~Hz}, 1 \mathrm{H}), 8.59 \sim 8.54(\mathrm{~m}, 3 \mathrm{H}), 7.77 \sim 7.74(\mathrm{~m}, 1 \mathrm{H})$, $7.67 \sim 7.57(\mathrm{~m}, 2 \mathrm{H}), 7.51 \sim 7.48(\mathrm{~m}, 1 \mathrm{H}), 4.64 \sim 4.60(\mathrm{q}$, $J=7.2 \mathrm{~Hz}, 2 \mathrm{H}), 1.59 \sim 1.56(\mathrm{t}, J=7.1 \mathrm{~Hz}, 3 \mathrm{H}) ;{ }^{13} \mathrm{C} \mathrm{NMR}$ $\left(126 \mathrm{MHz}, \mathrm{CDCl}_{3}\right) \delta: 172.78,162.63,133.52,130.21$, $129.34,127.40,126.63,125.91,125.84,125.11,124.81$, $124.02,122.67,122.25,101.39,61.86,14.21$; MS (ESI) $m / z: 289.1[\mathrm{M}+\mathrm{Na}]^{+}$.

10-差基-7-甲基菲-9-羧酸乙酯(2b): $64.5 \mathrm{mg}$, 产率 $77 \%$. 白色固体, m.p. 101 102 ${ }^{\circ} \mathrm{C}$ (文献值 ${ }^{[20]} 106 \sim$ $\left.107{ }^{\circ} \mathrm{C}\right) ;{ }^{1} \mathrm{H}$ NMR $\left(500 \mathrm{MHz}, \mathrm{CDCl}_{3}\right) \delta: 13.28(\mathrm{~s}, 1 \mathrm{H})$, $8.61(\mathrm{~s}, 1 \mathrm{H}), 8.56 \sim 8.52(\mathrm{~m}, 2 \mathrm{H}), 8.46 \sim 8.44(\mathrm{~d}, J=8.4$ $\mathrm{Hz}, 2 \mathrm{H}), 7.77 \sim 7.73(\mathrm{~m}, 1 \mathrm{H}), 7.63 \sim 7.60(\mathrm{~m}, 1 \mathrm{H}), 7.33 \sim$ $7.31(\mathrm{~m}, 1 \mathrm{H}), 4.64 \sim 4.60(\mathrm{q}, J=7.3 \mathrm{~Hz}, 2 \mathrm{H}), 2.56(\mathrm{~s}, 3 \mathrm{H})$, $1.60 \sim 1.57(\mathrm{t}, J=7.1 \mathrm{~Hz}, 3 \mathrm{H}) ;{ }^{13} \mathrm{C} \mathrm{NMR}(126 \mathrm{MHz}$, $\left.\mathrm{CDCl}_{3}\right) \delta: 172.87,162.69,137.20,133.72,130.29,129.53$, $126.33,125.95,125.67,124.90,124.86,123.87,122.69$, 122.19, 101.37, 61.91, 22.11, 14.25; MS (ESI) $\mathrm{m} / \mathrm{z}: 303.1$ $[\mathrm{M}+\mathrm{Na}]^{+}$. 10-羟基-7-甲氧基菲-9-羧酸乙酯(2c)：69.4 mg，产 率 78\%. 白色固体, m.p. 144 $145{ }^{\circ} \mathrm{C}$ (文献值 ${ }^{[20]} 138 \sim$ $\left.139{ }^{\circ} \mathrm{C}\right) ;{ }^{1} \mathrm{H} \mathrm{NMR}\left(500 \mathrm{MHz}, \mathrm{CDCl}_{3}\right) \delta: 13.46(\mathrm{~s}, 1 \mathrm{H})$, $8.52 \sim 8.50(\mathrm{~m}, 1 \mathrm{H}), 8.45 \sim 8.44(\mathrm{~d}, J=8.9 \mathrm{~Hz}, 2 \mathrm{H})$, $8.32 \sim 8.31(\mathrm{~d}, J=2.6 \mathrm{~Hz}, 1 \mathrm{H}), 7.74 \sim 7.71(\mathrm{~m}, 1 \mathrm{H})$, $7.58 \sim 7.55(\mathrm{~m}, 1 \mathrm{H}), 7.11 \sim 7.09(\mathrm{~m}, 1 \mathrm{H}), 4.62 \sim 4.57(\mathrm{q}$, $J=7.1 \mathrm{~Hz}, 2 \mathrm{H}), 3.94(\mathrm{~s}, 3 \mathrm{H}), 1.58 \sim 1.56(\mathrm{t}, J=7.1 \mathrm{~Hz}$, $3 \mathrm{H}) ;{ }^{13} \mathrm{C} \mathrm{NMR}\left(126 \mathrm{MHz}, \mathrm{CDCl}_{3}\right) \delta: 172.95,163.62$, $159.05,133.84,130.97,130.49,125.74,124.99,124.23$, $124.16,121.89,120.20,113.54,108.06,101.13,61.90$, 55.11, 14.31; MS (ESI) $m / z: 319.1[\mathrm{M}+\mathrm{Na}]^{+}$.

10-羟基-7-叔丁基菲-9-羧酸乙酯(2d)：80.7 mg，产 率 $83 \%$. 白色固体, m.p. $127 \sim 128{ }^{\circ} \mathrm{C}$ (文献值 ${ }^{[20]} 130 \sim$ $\left.131{ }^{\circ} \mathrm{C}\right) ;{ }^{1} \mathrm{H}$ NMR $\left(500 \mathrm{MHz}, \mathrm{CDCl}_{3}\right) \delta: 13.43$ (d, $J=1.2$ $\mathrm{Hz}, 1 \mathrm{H}), 8.92(\mathrm{~d}, J=1.9 \mathrm{~Hz}, 1 \mathrm{H}), 8.59 \sim 8.51(\mathrm{~m}, 3 \mathrm{H})$, $7.78 \sim 7.75(\mathrm{~m}, 1 \mathrm{H}), 7.65 \sim 7.58(\mathrm{~m}, 2 \mathrm{H}), 4.66 \sim 4.62(\mathrm{q}$, $J=7.1 \mathrm{~Hz}, 2 \mathrm{H}), 1.64 \sim 1.61(\mathrm{t}, J=7.1 \mathrm{~Hz}, 3 \mathrm{H}), 1.51(\mathrm{~s}$, $9 \mathrm{H}) ;{ }^{13} \mathrm{C} \mathrm{NMR}\left(126 \mathrm{MHz}, \mathrm{CDCl}_{3}\right) \delta: 173.14,162.89$, $150.22,133.61,130.31,129.24,126.37,124.99,124.81$, $123.82,122.53,122.31,122.21,101.66,61.88,35.18$, 31.46, 14.38; MS (ESI) $m / z: 345.1[\mathrm{M}+\mathrm{Na}]^{+}$.

10-羟基-7-正丁基菲-9-羧酸乙酯(2e): $86.7 \mathrm{mg}$ ，产 率 90\%. 白色固体, m.p. $61 \sim 62{ }^{\circ} \mathrm{C}$ (文献值 ${ }^{[20]} 63 \sim$ $\left.64{ }^{\circ} \mathrm{C}\right) ;{ }^{1} \mathrm{H}$ NMR $\left(500 \mathrm{MHz}, \mathrm{CDCl}_{3}\right) \delta: 13.32(\mathrm{~s}, 1 \mathrm{H}), 8.65$ $(\mathrm{s}, 1 \mathrm{H}), 8.57 \sim 8.53(\mathrm{~m}, 2 \mathrm{H}), 8.48 \sim 8.47(\mathrm{~d}, J=8.4 \mathrm{~Hz}$, $1 \mathrm{H}), 7.77 \sim 7.73(\mathrm{~m}, 1 \mathrm{H}), 7.64 \sim 7.61(\mathrm{~m}, 1 \mathrm{H}), 7.35 \sim 7.33$ $(\mathrm{m}, 1 \mathrm{H}), 4.65 \sim 4.60(\mathrm{q}, J=7.1 \mathrm{~Hz}, 2 \mathrm{H}), 2.85 \sim 2.82(\mathrm{t}, J=$ $7.6 \mathrm{~Hz}, 2 \mathrm{H}), 1.81 \sim 1.75(\mathrm{~m}, 2 \mathrm{H}), 1.61 \sim 1.58(\mathrm{t}, J=7.1$ $\mathrm{Hz}, 3 \mathrm{H}), 1.51 \sim 1.46(\mathrm{q}, J=7.4 \mathrm{~Hz}, 2 \mathrm{H}), 1.05 \sim 1.02(\mathrm{t}, J=$ $7.3 \mathrm{~Hz}, 3 \mathrm{H}) ;{ }^{13} \mathrm{C} \mathrm{NMR}\left(126 \mathrm{MHz}, \mathrm{CDCl}_{3}\right) \delta: 172.91$, $162.70,142.05,133.72,130.24,129.46,126.27,125.34$, $125.05,124.88,124.85,124.05,122.63,122.17,101.43$, $61.84,36.06,33.39,22.35,14.20,13.97$; MS (ESI) $\mathrm{m} / z$ : $345.1[\mathrm{M}+\mathrm{Na}]^{+}$.

10-差基-5-甲基菲-9-羧酸乙酯(2f)：39.2 mg，产率 $47 \%$. 淡黄色固体, m.p. $52 \sim 53{ }^{\circ} \mathrm{C}$ (文献值 ${ }^{[20]} 53 \sim$ $\left.54{ }^{\circ} \mathrm{C}\right) ;{ }^{1} \mathrm{H}$ NMR $\left(500 \mathrm{MHz}, \mathrm{CDCl}_{3}\right) \delta: 12.91(\mathrm{~s}, 1 \mathrm{H})$, $8.66 \sim 8.61(\mathrm{~m}, 3 \mathrm{H}), 7.75 \sim 7.72(\mathrm{~m}, 1 \mathrm{H}), 7.67 \sim 7.64(\mathrm{~m}$, $1 \mathrm{H}), 7.49 \sim 7.45$ (t, $J=7.8 \mathrm{~Hz}, 1 \mathrm{H}), 7.38 \sim 7.37$ (d, $J=7.1$ $\mathrm{Hz}, 1 \mathrm{H}), 4.64 \sim 4.60(\mathrm{q}, J=7.2 \mathrm{~Hz}, 2 \mathrm{H}), 3.05(\mathrm{~s}, 3 \mathrm{H})$, $1.57 \sim 1.54(\mathrm{t}, J=7.2 \mathrm{~Hz}, 3 \mathrm{H}) ;{ }^{13} \mathrm{C}$ NMR $(126 \mathrm{MHz}$, $\left.\mathrm{CDCl}_{3}\right) \delta: 172.59,161.32,135.07,134.37,130.78,129.02$, $128.73,127.61,126.57,126.43,126.33,126.16,124.47$, $123.85,102.57,61.98,27.19,14.34$; MS (ESI) $m / z: 303.1$ $[\mathrm{M}+\mathrm{Na}]^{+}$. 
10-羟基-5-甲氧基菲-9-羧酸乙酯 $(\mathbf{2 g})$ ： $82.9 \mathrm{mg}$, 产 率 47\%. 白色固体, m.p. 101 $102{ }^{\circ} \mathrm{C}$ (文献值 ${ }^{[20]} 100 \sim$ $\left.101{ }^{\circ} \mathrm{C}\right) ;{ }^{1} \mathrm{H}$ NMR $\left(500 \mathrm{MHz}, \mathrm{CDCl}_{3}\right) \delta: 13.06(\mathrm{~s}, 1 \mathrm{H})$, $9.62 \sim 9.61(\mathrm{~d}, J=8.7 \mathrm{~Hz}, 1 \mathrm{H}), 8.66 \sim 8.64(\mathrm{~d}, J=8.2 \mathrm{~Hz}$, $1 \mathrm{H}), 8.41 \sim 8.40(\mathrm{~d}, J=8.7 \mathrm{~Hz}, 1 \mathrm{H}), 7.80 \sim 7.77(\mathrm{~m}, 1 \mathrm{H})$, $7.68 \sim 7.65(\mathrm{t}, J=7.5 \mathrm{~Hz}, 1 \mathrm{H}), 7.53 \sim 7.49(\mathrm{t}, J=8.2 \mathrm{~Hz}$, $1 \mathrm{H}), 7.05 \sim 7.03(\mathrm{~d}, J=7.7 \mathrm{~Hz}, 1 \mathrm{H}), 4.64 \sim 4.60$ (q, $J=7.2$ $\mathrm{Hz}, 2 \mathrm{H}), 4.09$ (s, 3H), $1.57 \sim 1.54(\mathrm{t}, J=7.1 \mathrm{~Hz}, 3 \mathrm{H}) ;{ }^{13} \mathrm{C}$ NMR $\left(126 \mathrm{MHz}, \mathrm{CDCl}_{3}\right) \delta: 172.60,161.97,158.52$, $133.21,131.72,129.84,128.43,127.07,126.02,125.40$, 124.06, 118.50, 116.77, 106.65, 102.01, 61.93, 55.56, 14.23; MS (ESI) $m / z: 319.1[\mathrm{M}+\mathrm{Na}]^{+}$.

10-羟基-5-异丙基菲-9-羧酸乙酯(2h): $57.9 \mathrm{mg}$, 产 率 63\%. 淡黄色固体, m.p. 63 64 ${ }^{\circ} \mathrm{C}$ (文献值 ${ }^{[20]} 55 \sim$ $\left.56{ }^{\circ} \mathrm{C}\right) ;{ }^{1} \mathrm{H}$ NMR $\left(500 \mathrm{MHz}, \mathrm{CDCl}_{3}\right) \delta: 13.05(\mathrm{~s}, 1 \mathrm{H})$, $8.62 \sim 8.57(\mathrm{~m}, 2 \mathrm{H}), 8.35 \sim 8.33(\mathrm{~d}, J=8.2 \mathrm{~Hz}, 1 \mathrm{H})$, $7.71 \sim 7.63(\mathrm{~m}, 2 \mathrm{H}), 7.58 \sim 7.54(\mathrm{~m}, 2 \mathrm{H}), 4.64 \sim 4.59(\mathrm{q}$, $J=7.1 \mathrm{~Hz}, 2 \mathrm{H}), 4.17 \sim 4.12(\mathrm{~m}, 1 \mathrm{H}), 1.57 \sim 1.54(\mathrm{t}, J=7.1$ $\mathrm{Hz}, 3 \mathrm{H}), 1.48 \sim 1.47(\mathrm{~d}, J=6.7 \mathrm{~Hz}, 6 \mathrm{H}) ;{ }^{13} \mathrm{C}$ NMR $(126$ $\left.\mathrm{MHz}, \mathrm{CDCl}_{3}\right) \delta: 172.70,161.64,146.53,133.37,130.19$, $128.37,127.58,126.76,126.61,126.39,126.26,125.77$, 124.34, 123.50, 122.73, 102.25, 61.94, 31.35, 25.60, 14.34; MS (ESI) $m / z: 331.1[\mathrm{M}+\mathrm{Na}]^{+}$.

9-羟基菲-2,10-二甲酸二乙酯 $(\mathbf{2 i})$ : $78.0 \mathrm{mg}$, 产率 $77 \%$. 白色固体, m.p. 131 132 ${ }^{\circ} \mathrm{C}$ (文献值 ${ }^{[20]} 135 \sim$ $\left.136{ }^{\circ} \mathrm{C}\right) ;{ }^{1} \mathrm{H}$ NMR (500 MHz, $\left.\mathrm{CDCl}_{3}\right) \delta: 13.45$ (s, 1H), $9.56 \sim 9.56(\mathrm{~d}, J=1.5 \mathrm{~Hz}, 1 \mathrm{H}), 8.56 \sim 8.53(\mathrm{~m}, 3 \mathrm{H})$, $8.08 \sim 8.06(\mathrm{~m}, 1 \mathrm{H}), 7.80 \sim 7.77(\mathrm{~m}, 1 \mathrm{H}), 7.70 \sim 7.67(\mathrm{~m}$, $1 \mathrm{H}), 4.64 \sim 4.60(\mathrm{q}, J=7.2 \mathrm{~Hz}, 2 \mathrm{H}), 4.49 \sim 4.44$ (q, $J=7.1$ $\mathrm{Hz}, 2 \mathrm{H}), 1.64 \sim 1.62(\mathrm{t}, J=7.1 \mathrm{~Hz}, 3 \mathrm{H}), 1.49 \sim 1.46(\mathrm{t}, J=$ $7.2 \mathrm{~Hz}, 3 \mathrm{H}) ;{ }^{13} \mathrm{C} \mathrm{NMR}\left(126 \mathrm{MHz}, \mathrm{CDCl}_{3}\right) \delta: 172.65$, $166.95,163.24,132.85,130.62,129.00,128.91,128.23$, $127.79,126.07,125.08,124.27,123.00,122.82,101.42$, 101.42, 62.29, 61.03, 14.45, 14.17; MS (ESI) $\mathrm{m} / \mathrm{z}: 361.1$ $[\mathrm{M}+\mathrm{Na}]^{+}$.

10-羟基-7-乙烯基菲-9-羧酸乙酯 $(2 \mathbf{j}): 26.0 \mathrm{mg}$, 产率 $30 \%$. 白色固体, m.p. $104 \sim 105{ }^{\circ} \mathrm{C}$ (文献值 ${ }^{[20]} 113 \sim$ $\left.114{ }^{\circ} \mathrm{C}\right) ;{ }^{1} \mathrm{H} \mathrm{NMR}\left(500 \mathrm{MHz}, \mathrm{CDCl}_{3}\right) \delta: 13.38$ (s, 1H), $8.84(\mathrm{~s}, 1 \mathrm{H}), 8.54 \sim 8.47(\mathrm{~m}, 3 \mathrm{H}), 7.77 \sim 7.74(\mathrm{~m}, 1 \mathrm{H})$, $7.64 \sim 7.56(\mathrm{~m}, 2 \mathrm{H}), 6.93 \sim 6.87(\mathrm{dd}, J=17.6,10.8 \mathrm{~Hz}$, $1 \mathrm{H}), 5.93 \sim 5.90(\mathrm{~d}, J=17.8 \mathrm{~Hz}, 1 \mathrm{H}), 5.39 \sim 5.37(\mathrm{~d}, J=$ $11.0 \mathrm{~Hz}, 1 \mathrm{H}), 4.64 \sim 4.59(\mathrm{q}, J=7.1 \mathrm{~Hz}, 2 \mathrm{H}), 1.61 \sim 1.58$ $(\mathrm{t}, J=7.1 \mathrm{~Hz}, 3 \mathrm{H}) ;{ }^{13} \mathrm{C} \mathrm{NMR}\left(126 \mathrm{MHz}, \mathrm{CDCl}_{3}\right) \delta: 172.87$, $163.05,137.47,136.56,133.50,130.49,129.66,126.81$, $125.70,125.22$, 125.02, 124.46, 123.09, 122.45, 121.87,
114.21, 101.46, 62.05, 14.31; MS (ESI) m/z: $315.1[\mathrm{M}+$ $\mathrm{Na}]^{+}$.

7-氯-10-羟基菲-9-羧酸乙酯 $(2 \mathbf{k})$ : $63.1 \mathrm{mg}$, 产率 $70 \%$. 白色固体, m.p. $121 \sim 122{ }^{\circ} \mathrm{C}$ (文献值 ${ }^{[20]} 125 \sim$ $\left.126{ }^{\circ} \mathrm{C}\right) ;{ }^{1} \mathrm{H}$ NMR $\left(500 \mathrm{MHz}, \mathrm{CDCl}_{3}\right) \delta: 13.46(\mathrm{~s}, 1 \mathrm{H})$, $8.74 \sim 8.73(\mathrm{~d}, J=2.2 \mathrm{~Hz}, 1 \mathrm{H}), 8.47 \sim 8.46(\mathrm{~m}, 1 \mathrm{H})$, $8.38 \sim 8.32(\mathrm{~m}, 2 \mathrm{H}), 7.73 \sim 7.70(\mathrm{~m}, 1 \mathrm{H}), 7.62 \sim 7.59(\mathrm{~m}$, $1 \mathrm{H}), 7.37 \sim 7.34(\mathrm{~m}, 1 \mathrm{H}), 4.62 \sim 4.57(\mathrm{q}, J=7.1 \mathrm{~Hz}, 2 \mathrm{H})$, $1.59 \sim 1.57(\mathrm{t}, J=7.1 \mathrm{~Hz}, 3 \mathrm{H}) ;{ }^{13} \mathrm{C}$ NMR $(126 \mathrm{MHz}$, $\left.\mathrm{CDCl}_{3}\right) \delta: 172.46,163.67,133.63,133.01,130.67,130.53$, $126.99,125.47,125.03,124.34,124.26,124.09,122.23$, 100.54, 62.29, 14.21; MS (ESI) $m / z: 323.0[\mathrm{M}+\mathrm{Na}]^{+}$.

7-氟-10-羟基菲-9-羧酸乙酯(2l): $54.7 \mathrm{mg}$, 产率 64\%. 白色固体, m.p. $122 \sim 123{ }^{\circ} \mathrm{C}$ (文献值 ${ }^{[20]} 125 \sim$ $\left.126{ }^{\circ} \mathrm{C}\right) ;{ }^{1} \mathrm{H} \mathrm{NMR}\left(500 \mathrm{MHz}, \mathrm{CDCl}_{3}\right) \delta: 13.53(\mathrm{~s}, 1 \mathrm{H})$, $8.51 \sim 8.40(\mathrm{~m}, 4 \mathrm{H}), 7.75 \sim 7.72(\mathrm{~m}, 1 \mathrm{H}), 7.62 \sim 7.59(\mathrm{~m}$, $1 \mathrm{H}), 7.20 \sim 7.17(\mathrm{~m}, 1 \mathrm{H}), 4.64 \sim 4.59(\mathrm{q}, J=7.1 \mathrm{~Hz}, 2 \mathrm{H})$, $1.59 \sim 1.57(\mathrm{t}, J=7.1 \mathrm{~Hz}, 3 \mathrm{H}) ;{ }^{13} \mathrm{C}$ NMR $(126 \mathrm{MHz}$, $\left.\mathrm{CDCl}_{3}\right) \delta: 172.61,163.95,163.14,161.20,133.25,131.16$, $131.07,130.66,126.52,125.03,124.77,124.69,122.48$, $122.14,112.52,112.34,111.51,111.31,100.89,100.87$, 62.23, 14.25; MS (ESI) $m / z: 307.1[\mathrm{M}+\mathrm{Na}]^{+}$.

10-羟基-6-甲基菲-9-羧酸乙酯/10-羟基-8-甲基菲-9羧酸乙酯 $=4 / 1(2 \mathrm{~m})^{[20]}: 40.0 \mathrm{mg}$, 产率 $48 \%$. 白色固体, m.p. $88 \sim 89{ }^{\circ} \mathrm{C} ;{ }^{1} \mathrm{H}$ NMR $\left(500 \mathrm{MHz}, \mathrm{CDCl}_{3}\right) \delta$ : $13.28(\mathrm{~s}$, $1 \mathrm{H}), 10.92(\mathrm{~s}, 0.25 \mathrm{H}), 8.70 \sim 8.68(\mathrm{~d}, J=8.7 \mathrm{~Hz}, 1 \mathrm{H})$, $8.58 \sim 8.55(\mathrm{~m}, 1.8 \mathrm{H}), 8.58 \sim 8.55(\mathrm{~m}, 0.45 \mathrm{H}), 8.49 \sim 8.47$ $(\mathrm{m}, 0.25 \mathrm{H}), 8.43 \sim 8.41(\mathrm{~m}, 0.25 \mathrm{H}), 8.36(\mathrm{~s}, 1 \mathrm{H}), 7.78 \sim$ $7.73(\mathrm{~m}, 1 \mathrm{H}), 7.78 \sim 7.73(\mathrm{~m}, 0.25 \mathrm{H}), 7.66 \sim 7.62(\mathrm{~m}, 1 \mathrm{H})$, $7.66 \sim 7.62(\mathrm{~m}, 0.25 \mathrm{H}), 7.47 \sim 7.40(\mathrm{~m}, 1.2 \mathrm{H}), 7.47 \sim 7.40$ $(\mathrm{m}, 0.3 \mathrm{H}), 4.64 \sim 4.60(\mathrm{q}, J=7.1 \mathrm{~Hz}, 2 \mathrm{H}), 4.50 \sim 4.46(\mathrm{q}$, $J=7.2 \mathrm{~Hz}, 0.5 \mathrm{H}), 2.58(\mathrm{~s}, 3 \mathrm{H}), 2.55(\mathrm{~s}, 0.75 \mathrm{H}), 1.59 \sim 1.56$ $(\mathrm{t}, J=7.1 \mathrm{~Hz}, 3 \mathrm{H}), 1.41 \sim 1.38(\mathrm{t}, J=7.2 \mathrm{~Hz}, 0.75 \mathrm{H}) ;{ }^{13} \mathrm{C}$ NMR $\left(126 \mathrm{MHz}, \mathrm{CDCl}_{3}\right) \delta: 172.92,171.69,162.06$, $158.22,134.15,133.75,133.59,133.44,130.42,130.20$, $129.98,129.15,128.78,127.20,127.12,126.82,126.69$, $126.15,125.88,125.35,124.93,124.52,124.51,122.72$, $122.39,120.27,103.64,101.54,61.92,61.74,22.92,21.45$, 14.34, 14.11; MS (ESI) $m / z: 303.1[\mathrm{M}+\mathrm{Na}]^{+}$.

10-羟基-7-甲硫基菲-9-羧酸乙酯(2n)：28.5 mg，产 率 30\%. 白色固体, m.p. 141 142 ${ }^{\circ} \mathrm{C} ;{ }^{1} \mathrm{H}$ NMR (500 $\left.\mathrm{MHz}, \mathrm{CDCl}_{3}\right) \delta: 13.46(\mathrm{~s}, 1 \mathrm{H}), 8.66 \sim 8.65(\mathrm{~d}, J=11.8 \mathrm{~Hz}$, $1 \mathrm{H}), 8.53 \sim 8.48(\mathrm{~m}, 2 \mathrm{H}), 8.43 \sim 8.42(\mathrm{~d}, J=8.7 \mathrm{~Hz}, 1 \mathrm{H})$, $7.76 \sim 7.73(\mathrm{~m}, 1 \mathrm{H}), 7.62 \sim 7.59(\mathrm{~m}, 1 \mathrm{H}), 7.37 \sim 7.35(\mathrm{~m}$, $1 \mathrm{H}), 4.63 \sim 4.59$ (q, $J=7.2 \mathrm{~Hz}, 2 \mathrm{H}), 2.62(\mathrm{~s}, 3 \mathrm{H}), 1.59 \sim$ 
$1.56(\mathrm{t}, J=7.1 \mathrm{~Hz}, 3 \mathrm{H}) ;{ }^{13} \mathrm{C}$ NMR $\left(126 \mathrm{MHz}, \mathrm{CDCl}_{3}\right) \delta$ : $172.85,163.47,138.18,133.52,130.55,129.96,126.48$, $125.00,124.77,123.32,123.09,122.80,122.20,122.08$, 100.82, 62.08, 15.57, 14.39; MS (ESI) $m / z: 335.1[\mathrm{M}+$ $\mathrm{Na}]^{+}$; HRMS (ESI) calcd for $\mathrm{C}_{18} \mathrm{H}_{16} \mathrm{O}_{3} \mathrm{~S}[\mathrm{M}+\mathrm{Na}]^{+}$ 335.0718 , found 335.0712 .

5-氟-10-羟基菲-9-羧酸乙酯(2o): $50.3 \mathrm{mg}$, 产率 $59 \%$. 白色固体, m.p. 89 90 ${ }^{\circ} \mathrm{C}$ (文献值 ${ }^{[20]} 93 \sim 94{ }^{\circ} \mathrm{C}$ ); ${ }^{1} \mathrm{H}$ NMR $\left(500 \mathrm{MHz}, \mathrm{CDCl}_{3}\right) \delta: 13.26(\mathrm{~s}, 1 \mathrm{H}), 9.03 \sim 9.01$ $(\mathrm{m}, 1 \mathrm{H}), 8.60 \sim 8.54(\mathrm{~m}, 2 \mathrm{H}), 7.80 \sim 7.77(\mathrm{~m}, 1 \mathrm{H}), 7.68 \sim$ $7.65(\mathrm{t}, J=7.5 \mathrm{~Hz}, 1 \mathrm{H}), 7.49 \sim 7.44(\mathrm{~m}, 1 \mathrm{H}), 7.22 \sim 7.17$ (m, $1 \mathrm{H}), 4.63 \sim 4.59$ (q, $J=7.2 \mathrm{~Hz}, 2 \mathrm{H}), 1.57 \sim 1.54(\mathrm{t}, J=$ $7.1 \mathrm{~Hz}, 3 \mathrm{H}) ;{ }^{13} \mathrm{C}$ NMR $\left(126 \mathrm{MHz}, \mathrm{CDCl}_{3}\right) \delta: 172.51$, $162.83,162.53,160.53,132.00,131.97,131.36,131.31$, $130.75,130.73,127.58,127.38,127.35,127.29,127.03$, 127.02, 125.64, 124.61, 121.67, 121.64, 115.67, 115.60, 111.46, 111.26, 101.49, 101.46, 62.22, 14.30; MS (ESI) $\mathrm{m} / \mathrm{z}: 307.1[\mathrm{M}+\mathrm{Na}]^{+}$.

10-羊基-6,8-二甲基菲-9-羧酸乙酯(2p): $37.6 \mathrm{mg}$, 产 率 $43 \%$. 淡黄色固体, m.p. $100 \sim 101{ }^{\circ} \mathrm{C}$ (文献值 ${ }^{[20]}$ $\left.119 \sim 120{ }^{\circ} \mathrm{C}\right) ;{ }^{1} \mathrm{H}$ NMR $\left(500 \mathrm{MHz}, \mathrm{CDCl}_{3}\right) \delta: 10.79$ (s, $1 \mathrm{H}), 8.54 \sim 8.53(\mathrm{~d}, J=8.3 \mathrm{~Hz}, 1 \mathrm{H}), 8.43 \sim 8.41(\mathrm{~m}, 1 \mathrm{H})$, $8.19(\mathrm{~s}, 1 \mathrm{H}), 7.71 \sim 7.68(\mathrm{~m}, 1 \mathrm{H}), 7.60 \sim 7.57(\mathrm{~m}, 1 \mathrm{H})$, $7.23(\mathrm{~s}, 1 \mathrm{H}), 4.45 \sim 4.40$ (q, $J=7.1 \mathrm{~Hz}, 2 \mathrm{H}), 2.51(\mathrm{~s}, 3 \mathrm{H})$, 2.47 (s, 3H), $1.35 \sim 1.33$ (t, $J=7.2 \mathrm{~Hz}, 3 \mathrm{H}) ;{ }^{13} \mathrm{C}$ NMR $(126$ $\left.\mathrm{MHz}, \mathrm{CDCl}_{3}\right) \delta: 171.74,157.61,133.94,133.54,132.03$, $129.81,127.31,126.70,126.53,124.66,124.49,122.73$, 120.32, 103.65, 61.69, 22.77, 21.43, 14.12; MS (ESI) $\mathrm{m} / \mathrm{z}$ : $317.1[\mathrm{M}+\mathrm{Na}]^{+}$.

5-羟基四苯-6-羧酸乙酯 $(2 q)$ ): $48.2 \mathrm{mg}$, 产率 $51 \%$. 淡黄色固体, m.p. $124 \sim 125{ }^{\circ} \mathrm{C}$ (文献值 ${ }^{[20]} 103 \sim 104{ }^{\circ} \mathrm{C}$ ); ${ }^{1} \mathrm{H}$ NMR $\left(500 \mathrm{MHz}, \mathrm{CDCl}_{3}\right) \delta: 11.15(\mathrm{~s}, 1 \mathrm{H}), 8.64 \sim 8.63$ (d, $J=8.3 \mathrm{~Hz}, 1 \mathrm{H}), 8.58 \sim 8.51(\mathrm{~m}, 2 \mathrm{H}), 8.03 \sim 8.01(\mathrm{~d}, J$ $=8.4 \mathrm{~Hz}, 1 \mathrm{H}), 7.94 \sim 7.93(\mathrm{~d}, J=7.8 \mathrm{~Hz}, 1 \mathrm{H}), 7.89 \sim 7.88$ (d, $J=8.8 \mathrm{~Hz}, 1 \mathrm{H}), 7.82 \sim 7.79(\mathrm{~m}, 1 \mathrm{H}), 7.70 \sim 7.67(\mathrm{~m}$, $1 \mathrm{H}), 7.58 \sim 7.48(\mathrm{~m}, 1 \mathrm{H}), 4.40 \sim 4.36(\mathrm{q}, \quad J=7.1 \mathrm{~Hz}, 2 \mathrm{H})$, $1.18 \sim 1.15(\mathrm{t}, J=7.1 \mathrm{~Hz}, 3 \mathrm{H}) ;{ }^{13} \mathrm{C}$ NMR $(126 \mathrm{MHz}$, $\left.\mathrm{CDCl}_{3}\right) \delta: 172.46,158.53,133.21,133.00,130.05,129.54$, $128.93,127.80,126.73,126.03,125.91,124.66,124.27$, $123.99,122.94,120.19,103.78,61.65,13.75$; MS (ESI) $m / z: 339.1[\mathrm{M}+\mathrm{Na}]^{+}$.

5-羟基苯并 $[c]$ 菲-6-羧酸乙酯(2r): $31.2 \mathrm{mg}$, 产率 $33 \%$. 淡黄色固体, m.p. 95 96 ${ }^{\circ} \mathrm{C}$ (文献值 ${ }^{[20]} 102 \sim$ $\left.103{ }^{\circ} \mathrm{C}\right) ;{ }^{1} \mathrm{H}$ NMR $\left(500 \mathrm{MHz}, \mathrm{CDCl}_{3}\right) \delta: 13.01(\mathrm{~s}, 1 \mathrm{H})$, $8.91 \sim 8.85(\mathrm{~m}, 2 \mathrm{H}), 8.78 \sim 8.76(\mathrm{~d}, J=9.1 \mathrm{~Hz}, 1 \mathrm{H})$,
$8.67 \sim 8.65(\mathrm{~m}, 1 \mathrm{H}), 7.99 \sim 7.97(\mathrm{~m}, 1 \mathrm{H}), 7.90 \sim 7.77(\mathrm{~m}$, $1 \mathrm{H}), 7.76 \sim 7.67(\mathrm{~m}, 1 \mathrm{H}), 7.66 \sim 7.56(\mathrm{~m}, 2 \mathrm{H}), 4.67 \sim 4.63$ $(\mathrm{q}, J=7.2 \mathrm{~Hz}, 2 \mathrm{H}), 1.59 \sim 1.56(\mathrm{t}, J=7.1 \mathrm{~Hz}, 3 \mathrm{H}) ;{ }^{13} \mathrm{C}$ NMR $\left(126 \mathrm{MHz}, \mathrm{CDCl}_{3}\right) \delta$ : 172.62, 161.70, 133.21, $131.76,129.93,129.35,128.44,128.34,127.91,127.85$, $127.50,126.20,125.95,125.69,125.29,124.51,123.97$, 122.74, 102.68, 62.18, 14.37; MS (ESI) $\mathrm{m} / z$ : $339.1[\mathrm{M}+$ $\mathrm{Na}]^{+}$.

10-差基- $N$-苯基菲-9-甲酰胺(2s)：29.8 mg, 产率 $32 \%$. 白色固体, m.p. $193 \sim 194{ }^{\circ} \mathrm{C}$ (文献值 ${ }^{[20]} 111 \sim$ $\left.112{ }^{\circ} \mathrm{C}\right) ;{ }^{1} \mathrm{H}$ NMR $\left(500 \mathrm{MHz}, \mathrm{DMSO}-d_{6}\right) \delta: 10.57(\mathrm{~s}, 1 \mathrm{H})$, $10.11(\mathrm{~s}, 1 \mathrm{H}), 8.88 \sim 8.86(\mathrm{~d}, J=8.0 \mathrm{~Hz}, 1 \mathrm{H}), 8.81 \sim 8.79$ $(\mathrm{d}, J=8.1 \mathrm{~Hz}, 1 \mathrm{H}), 8.43 \sim 8.41(\mathrm{~d}, J=8.2 \mathrm{~Hz}, 1 \mathrm{H}), 7.86 \sim$ $7.85(\mathrm{~d}, J=7.8 \mathrm{~Hz}, 2 \mathrm{H}), 7.80 \sim 7.72(\mathrm{~m}, 3 \mathrm{H}), 7.62 \sim 7.54$ (m, 2H), $7.40 \sim 7.37$ (t, $J=7.8 \mathrm{~Hz}, 2 \mathrm{H}), 7.14 \sim 7.11(\mathrm{t}, J=$ $7.4 \mathrm{~Hz}, 1 \mathrm{H}) ;{ }^{13} \mathrm{C}$ NMR (126 MHz, DMSO) $\delta: 165.99$, $147.19,139.58,130.95,130.00,128.66,127.87,127.40$, $126.78,126.10,125.52,124.33,124.19,123.45,123.17$, 123.01, 119.63, 116.73; MS (ESI) $m / z: 336.1[\mathrm{M}+\mathrm{Na}]^{+}$.

2-氟-10-羟基菲-9-羧酸乙酯(2t): $85.3 \mathrm{mg}$, 产率 $85 \%$. 白色固体, m.p. 94 95 ${ }^{\circ} \mathrm{C}$ (文献值 ${ }^{[20]} 97 \sim 98{ }^{\circ} \mathrm{C}$ ); ${ }^{1} \mathrm{H}$ NMR $\left(500 \mathrm{MHz}, \mathrm{CDCl}_{3}\right) \delta: 13.21(\mathrm{~s}, 1 \mathrm{H}), 8.75 \sim 8.74$ (d, $J=8.4 \mathrm{~Hz}, 1 \mathrm{H}), 8.47 \sim 8.39(\mathrm{~m}, 2 \mathrm{H}), 8.11 \sim 8.09(\mathrm{~m}$, $1 \mathrm{H}), 7.56 \sim 7.53(\mathrm{t}, J=7.7 \mathrm{~Hz}, 1 \mathrm{H}), 7.48 \sim 7.42(\mathrm{~m}, 2 \mathrm{H})$, $4.64 \sim 4.59(\mathrm{q}, J=7.1 \mathrm{~Hz}, 2 \mathrm{H}), 1.59 \sim 1.56(\mathrm{t}, J=7.1 \mathrm{~Hz}$, $3 \mathrm{H}) ;{ }^{13} \mathrm{C}$ NMR $\left(126 \mathrm{MHz}, \mathrm{CDCl}_{3}\right) \delta: 172.62,162.44$, $161.43,161.41,160.48,130.02,128.80,127.27,126.68$, $126.61,125.95,125.58,124.78,124.72,124.36,122.50$, 119.06, 118.87, 109.68, 109.50, 102.31, 62.14, 14.26; MS (ESI) $m / z: 307.1[\mathrm{M}+\mathrm{Na}]^{+}$.

3-氟-10-羟基菲-9-羧酸乙酯 $(\mathbf{2 u}): 67.6 \mathrm{mg}$, 产率 $79 \%$. 白色固体, m.p. $111 \sim 112{ }^{\circ} \mathrm{C}$ (文献值 ${ }^{[20]} 97 \sim$ $\left.98{ }^{\circ} \mathrm{C}\right) ;{ }^{1} \mathrm{H}$ NMR $\left(500 \mathrm{MHz}, \mathrm{CDCl}_{3}\right) \delta$ : 13.37 (s, 1H), $8.75 \sim 8.73(\mathrm{~d}, J=8.6 \mathrm{~Hz}, 1 \mathrm{H}), 8.48 \sim 8.45(\mathrm{~m}, 1 \mathrm{H})$, $8.31 \sim 8.29(\mathrm{~d}, \quad J=8.2 \mathrm{~Hz}, 1 \mathrm{H}), 8.06 \sim 8.04(\mathrm{~d}, J=11.1$ $\mathrm{Hz}, 1 \mathrm{H}), 7.57 \sim 7.54(\mathrm{t}, J=7.7 \mathrm{~Hz}, 1 \mathrm{H}), 7.45 \sim 7.42(\mathrm{t}, J=$ $7.6 \mathrm{~Hz}, 1 \mathrm{H}), 7.32 \sim 7.27(\mathrm{~m}, 1 \mathrm{H}), 4.63 \sim 4.58$ (q, $J=7.2$ $\mathrm{Hz}, 2 \mathrm{H}), 1.59 \sim 1.56(\mathrm{t}, J=7.2 \mathrm{~Hz}, 3 \mathrm{H}) ;{ }^{13} \mathrm{C}$ NMR $(126$ $\left.\mathrm{MHz}, \mathrm{CDCl}_{3}\right) \delta: 172.78,165.12,163.13,162.33,135.84$, $135.77,129.93,128.11,127.83,127.75,125.99,125.18$, $125.15,124.13,122.92,121.78,115.62,115.44,107.79$, 107.61, 100.88, 62.02, 14.29; MS (ESI) $m / z: 307.1[\mathrm{M}+$ $\mathrm{Na}]^{+}$.

1-氟-10-羟基菲-9-羧酸乙酯(2v): $48.3 \mathrm{mg}$, 产率 $57 \%$. 白色固体, m.p. $117 \sim 118{ }^{\circ} \mathrm{C}$ (文献值 ${ }^{[20]} 121 \sim$ 
$\left.122{ }^{\circ} \mathrm{C}\right) ;{ }^{1} \mathrm{H}$ NMR $\left(500 \mathrm{MHz}, \mathrm{CDCl}_{3}\right) \delta: 13.46 \sim 13.45(\mathrm{~d}$, $J=3.2 \mathrm{~Hz}, 1 \mathrm{H}), 8.70 \sim 8.68(\mathrm{~m}, 1 \mathrm{H}), 8.50 \sim 8.48(\mathrm{~d}, J=$ $8.1 \mathrm{~Hz}, 1 \mathrm{H}), 8.36 \sim 8.35(\mathrm{~d}, J=8.4 \mathrm{~Hz}, 1 \mathrm{H}), 7.69 \sim 7.64$ $(\mathrm{m}, 1 \mathrm{H}), 7.59 \sim 7.56(\mathrm{~m}, 1 \mathrm{H}), 7.49 \sim 7.46(\mathrm{~m}, 1 \mathrm{H}), 7.30 \sim$ $7.26(\mathrm{~m}, 1 \mathrm{H}), 4.64 \sim 4.60(\mathrm{q}, J=7.2 \mathrm{~Hz}, 2 \mathrm{H}), 1.57 \sim 1.54$ $(\mathrm{t}, J=7.2 \mathrm{~Hz}, 3 \mathrm{H}) ;{ }^{13} \mathrm{C} \mathrm{NMR}\left(126 \mathrm{MHz}, \mathrm{CDCl}_{3}\right) \delta: 172.52$, $162.16,162.12,160.08,136.18,130.66,130.58,129.57$, $128.09,125.76,125.21,124.45,123.31,118.45,118.41$, $114.71,114.65,113.91,113.72,102.63,102.61,62.13$, 14.26; MS (ESI) $m / z: 307.1[\mathrm{M}+\mathrm{Na}]^{+}$.

10-羟基-2-甲氧基菲-9-羧酸乙酯(2w): $30.7 \mathrm{mg}$, 产 率 35\%. 白色固体, m.p. 121 $122{ }^{\circ} \mathrm{C}$ (文献值 ${ }^{[20]} 111 \sim$ $\left.112{ }^{\circ} \mathrm{C}\right) ;{ }^{1} \mathrm{H} \mathrm{NMR}\left(500 \mathrm{MHz}, \mathrm{CDCl}_{3}\right) \delta: 13.35$ (s, 1H), $8.81 \sim 8.79(\mathrm{~m}, 1 \mathrm{H}), 8.50 \sim 8.48(\mathrm{~d}, J=9.2 \mathrm{~Hz}, 2 \mathrm{H}), 7.90$ $(\mathrm{d}, J=2.8 \mathrm{~Hz}, 1 \mathrm{H}), 7.55 \sim 7.47(\mathrm{~m}, 2 \mathrm{H}), 7.40 \sim 7.38(\mathrm{dd}$, $J=9.1,2.8 \mathrm{~Hz}, 1 \mathrm{H}), 4.65 \sim 4.61$ (q, $J=7.2 \mathrm{~Hz}, 2 \mathrm{H}), 4.01$ $(\mathrm{s}, 3 \mathrm{H}), 1.59 \sim 1.56(\mathrm{t}, J=7.1 \mathrm{~Hz}, 3 \mathrm{H}) ;{ }^{13} \mathrm{C} \mathrm{NMR}(126$ $\left.\mathrm{MHz}, \mathrm{CDCl}_{3}\right) \delta: 173.04,162.02,158.64,128.36,127.95$, $126.61,126.48,126.19,125.94,124.25,124.18,122.33$, $121.10,104.65,101.98,62.05,55.54,14.36$; MS (ESI) $m / z$ : $319.1[\mathrm{M}+\mathrm{Na}]^{+}$.

\section{3 克级规模实验}

在氮气保护下, 将化合物 1 (3 mmol)、 $\mathrm{K}_{2} \mathrm{HPO}_{4}(3.6$ $\mathrm{mmol}) 、 \mathrm{Na}_{2} \mathrm{~S}_{2} \mathrm{O}_{8}(0.36 \mathrm{mmol}) 、 \mathrm{KI}(0.15 \mathrm{mmol}, 5 \mathrm{~mol} \%)$ 、 $\operatorname{Ir}\left[\mathrm{dF}\left(\mathrm{CF}_{3}\right) \mathrm{ppy}\right]_{2}(\mathrm{dtbbpy}) \mathrm{PF}_{6}(0.03 \mathrm{mmol}, 1 \mathrm{~mol} \%)$ 、甲苯 $(25 \mathrm{~mL})$ 和乙醇 $(5 \mathrm{~mL})$ 分别加入 $50 \mathrm{~mL}$ 干燥的 Schlenk 反 应管. 将反应混合物放置在距离 $45 \mathrm{~W}$ Blue LED 灯约 5 $\mathrm{cm}$ 处, 并在室温下搅拌 $48 \mathrm{~h}$ 后, 用旋转蒸发仪除去溶 剂, 将残渣与硅胶混合干法上柱. 以石油醚/乙酸乙酯 $(V: V=20: 1)$ 为洗脱剂, 在硅胶 $(200 \sim 300$ 目)上进行 柱色谱纯化，以 $40 \%$ 的收率得到纯产物 $\mathbf{2 a}$.

\section{辅助材料(Supporting Information) 所合成目标化合 物的 ${ }^{1} \mathrm{H}$ NMR 和 ${ }^{13} \mathrm{C}$ NMR 谱. 这些材料可以免费从本刊 网站(http://sioc-journal.cn/)上下载.}

\section{References}

[1] (a) Floyd, A. J.; Dyke, S. F.; Ward, S. E. Chem. Rev. 1976, 76, 509. (b) Kovács, A.; Vasas, A.; Hohmann, J. Phytochemistry 2008, 69, 1084 .

(c) Narita, A.; Wang, X.; Feng, X.; Mullen, K. Chem. Soc. Rev. 2015, 44, 6616.

[2] (a) De Alvarenga, M. A.; Gottlieb, O. R.; Magalhaes, M. T. Phytochemistry 1976, 15, 844.

(b) Pettit, G. R.; Singh, S. B.; Niven, M. L.; Schmidt, J. M. Can. J. Chem. 1988, 66, 406.

(c) Cragg, G. M.; Newman, D. J. J. Nat. Prod. 2004, 67, 232.

[3] (a) Huffman, C. W.; Traxler, J. T.; Krbechek, L. O.; Riter, R. R.; Wagner, R. G. J. Med. Chem. 1971, 14, 90. (b) Colwell, W. T.; Brown, V.; Christie, P.; Lange, J.; Reece, C.; Yamamoto, K.; Henry, D. W. J. Med. Chem. 1972, 15, 771.

[4] Matsuda, H.; Morikawa, T.; Xie, H.; Yoshikawa, M. Planta Med. 2004, 70, 847.

[5] (a) Fisch, M. H.; Flick, B. H.; Arditti, J. Phytochemistry 1973, 12, 437.

(b) Yamaki, M.; Bai, L.; Inoue, K.; Takagi, S. Phytochemistry 1989, 28,3503 .

[6] (a) Mitsuhashi, R.; Suzuki, Y.; Yamanari, Y.; Mitamura, H.; Kambe, T.; Ikeda, N.; Okamoto, H.; Fujiwara, A.; Yamaji, M.; Kawasaki, N.; Maniwa, Y.; Kubozono, Y. Nature 2010, 464, 76.

(b) Lewis, F. D.; Burch, E. L. J. Phys. Chem. 1996, 100, 4055.

(c) Machado, A. M.; Munaro, M.; Martins, T. D.; Dávila, L. Y. A.; Giro, R.; Caldas, M. J.; Atvars, T. D. Z.; Akcelrud, L. C. Macromolecules 2006, 39, 3398.

(d) Matsuo, Y.; Sato, Y.; Hashiguchi, M.; Matsuo, K.; Nakamura, E. Adv. Funct. Mater. 2009, 19, 2224.

[7] (a) Yadav, A. K.; Ila, J.; Junjappa, H. Eur. J. Org. Chem. 2010, 2010,338

(b) Gupta, V.; Rao, V. U. B.; Das, T.; Vanka, K.; Singh, R. P. J. Org. Chem. 2016, 81, 5663.

(c) Gupta, V.; Pandey, S. K.; Singh, R. P. Org. Biomol. Chem. 2018 16,7134 .

[8] (a) Bogert, M. T. Science 1933, 77, 289.

(b) Floyd, A. J.; Dyke, S. F.; Ward, S. E. Chem. Rev. 1976, 76, 509.

[9] (a) Almeida, J. F.; Castedo, L.; Fernández, D.; Neo, A. G.; Romero, V.; Tojo, G. Org. Lett. 2003, 5, 4939.

(b) Kang, Y.; Wang, T.; Liang, Y.; Zhang, Y.; Wang, R.; Zhang, Z. RSC Adv. 2017, 7, 44333.

(c) Kang, T.; Zhang, W.; Wang, T.; Liang, Y.; Zhang, Z. J. Org. Chem. 2019, 84, 12387.

(d) Neo, A. G.; López, C.; Romero, V.; Antelo, B.; Delamano, J.; Pérez, A.; Fernandez, D.; Almeida, J. E.; Castedo, L.; Tojo, G. $J$. Org. Chem. 2010, 75, 6764.

[10] (a) Mallory, F. B.; Wood, C. S.; Gordon, J. T. J. Am. Chem. Soc. 1964, 86, 3094.

(b) Wood, C. S.; Mallory, F. B. J. Org. Chem. 1964, 29, 3373. (c) Matsushima, T.; Kobayashi, S.; Watanabe, S. J. Org. Chem. 2016, 81, 7799.

[11] Harrowven, D. C.; Nunn, M. I. T.; Fenwick, D. R. Tetrahedron Lett. 2002, 43, 3185 .

[12] Xia, Y.; Liu, Z.; Xiao, Q.; Qu, P.; Ge, R.; Zhang, Y.; Wang, J. Angew. Chem., Int. Ed. 2012, 51, 5714.

[13] (a) McMurry, J. E. Acc. Chem. Res. 1983, 16, 405.

(b) McMurry J. E. Chem. Rev. 1989, 89, 1513.

(c) Gies, A. E.; Pfeffer, M. J. Org. Chem. 1999, 64, 3650

[14] (a) Iuliano, A.; Piccioli, P.; Fabbri, D. Org. Lett. 2004, 6, 3711.

(b) Donohoe, T. J.; Orr, A. J.; Bingham, M. Angew. Chem., Int. Ed. 2006, 45, 2664.

(c) McAtee, C. C.; Riehl, P. S.; Schindler, C. S. J. Am. Chem. Soc. 2017, 139, 2960.

[15] Larock, R. C.; Doty, M. J.; Tian, Q.; Zenner, J. M. J. Org. Chem. 1997, 6, 7536

(b) Matsumoto, A.; Ilies, L.; Nakamura, E. J. Am. Chem. Soc. 2011, 133, 6557.

(c) Yan, J.; Yoshikai, N. Org. Lett. 2017, 19, 6630.

[16] Gou, B.; Yang, H.; Sun, H.; Chen, J.; Wu, J.; Zhou, L. Org. Lett. 2019, 21,80 .

(b) Yao, T.; Zhang, H.; Zhao, Y. Org. Lett. 2016, 18, 2532.

(c) Song, J.; Wang, S.; Sun, H.; Fan, Y.; Xiao, K.; Qian, Y. Org. Biomol. Chem. 2019, 17, 3328.

(d) Iwasaki, M.; Araki, Y.; Nishihara, Y. J. Org. Chem. 2017, 82, 6242.

[17] Liu, Y.; Chen, L.; Wang, Z.; Liu, P.; Liu, Y.; Dai, B. J. Org. Chem. 2019, 84, 204.

[18] (a) Liu, W.; Zhang, Y.; Guo, H. J. Org. Chem. 2018, 83, 10518.

(b) Yao, T.; Campo, M. A.; Larock, R. C. J. Org. Chem. 2005, 70, 3511. 
[19] (a) Xuan, J.; Xiao, W. Angew. Chem., Int. Ed. 2012, 51, 6828.

(b) Nicewicz, D. A.; MacMillan, D. W. C. Science 2008, 322, 77.

(c) Dai, C.; Narayanam, J. M. R.; Stephenson, C. R. J. Nat. Chem. 2011, 3, 140.

(d) Uygur, M.; Danelzik, T.; Mancheño, O. G. Chem. Commun. 2019, 55, 2980.

(e) Chen, J.; Cen, J.; Xu, X.; Li, X. Catal. Sci. Technol. 2016, 6, 349.

(f) Chen, Y.; Lu, L.; Yu, D.; Zhu, C.; Xiao, W. Sci. China Chem. 2019, 62, 24.

(g) Goddard, J. P.; Ollivier, C.; Fensterbank, L. Acc. Chem. Res. 2016, 49, 1924

(h) Yoon, T. P.; Ischay, M. A.; Du, J. Nat. Chem. 2010, $2,527$.

(i) Zhang, H.; Lei, A. Asian J. Org. Chem. 2018, 7, 1164.

(j) Yu, X.; Zhao, Q.; Chen, J.; Xiao, W.; Chen, J. Acc. Chem Res. $\mathbf{2 0 2 0}, 53,1066$.

(k) Yang, X.; Guo, J.; Xiao, H.; Feng, K.; Chen, B.; Tung, C.; Wu, L. Angew. Chem. Int. Ed. 2020, 59, 5365.

(1) Zhang, Q.; Xiong, Q.; Li, M.; Xiong, W.; Shi, B.; Lan, Y.; Lu, L.; Xiao, W. Angew. Chem. Int. Ed. 2020, DOI: 10.1002/anie. 202005313.

[20] Jiang, Y.; Yu, Z.; Zhang, Y.; Wang, B. Org. Lett. 2018, 20, 3728.

[21] (a) Dai, X.; Cheng, D.; Guan, B.; Mao, W.; Xu, X.; Li, X. J. Org.
Chem. 2014, 79, 7212 .

(b) Dai, X.; Mao, R.; Guan, B.; Xu, X.; Li, X. RSC Adv. 2015, 5, 55290 .

(c) Guan, B.; Xu, X.; Wang, H.; Li, X. Chin. J. Org. Chem. 2016, 36, 1564.

(d) Ye, Q.; Ye, H.; Cheng, D.; Li, X.; Xu, X. Tetrahedron Lett. 2018, 59, 2546.

(e) Ye, H.; Ye, Q.; Cheng, D.; Li, X.; Xu, X. Tetrahedron Lett. 2018, 59, 2046.

(f) Ye, H.; Zhao, H.; Ren, S.; Ye, H.; Cheng, D.; Li, X.; Xu, X. Tetrahedron Lett. 2019, 60, 1302.

[22] Becker, P.; Duhamel, T.; Stein, C. J.; Reiher, M.; Muñiz, K. Angew. Chem. Int. Ed. 2017, 56, 8004.

[23] Uyanik, M.; Hayashi, H.; Ishihara, K. Science 2014, 345, 291.

[24] (a) Fürstner, A.; Mamane, V. J. Org. Chem. 2002, 67, 6264.

(b) Jiang, Y.; Chen, X.; Zheng, Y.; Xue, Z.; Shu, C.; Yuan, W.; Zhang, X. Angew. Chem. Int. Ed. 2011, 50, 7304.

[25] Hsiao, Y.; Rivera, N. R.; Rosner, T.; Krska, S. W.; Njolito, E.; Wang, F.; Sun, Y.; Armstrong, J. D.; Grabowski, E. J. J.; Tillyer, R. D.; Spindler, F.; Malan, C. J. Am. Chem. Soc. 2004, 126, 9918.

[26] Ji, Y.; Trenkle, W. C.; Vowles, J. V. Org. Lett. 2006, 8, 1161.

[27] Jiang, J.; Wang, Y.; Zhang, X. ACS Catal. 2014, 4, 1570.

(Li, L.; Fan, Y.) 